\title{
Enormous Hyper-Rayleigh Scattering from Nanocrystalline Gold Particle Suspensions
}

\author{
Fredrick W. Vance, Buford I. Lemon, and Joseph T. Hupp* \\ Department of Chemistry and Materials Research Center, Northwestern University, Evanston, Illinois 60208
}

Received: October 12, 1998

\begin{abstract}
The recent emergence of advanced technological applications for colloidal gold suspensions and related particle assemblies and interfaces has created a demand for new chemical and physical techniques with which to characterize them. For macroscopic samples/interfaces, coherent second harmonic generation (SHG) has proven itself a useful characterization tool due, at least in part, to metal-based plasmon enhancement. In an effort to defeat or bypass the size restrictions inherent to SHG, we have utilized a related incoherent methodology, hyper-Rayleigh scattering (HRS), to interrogate aqueous colloidal suspensions of $13 \mathrm{~nm}$ diameter gold particles. The nanoscale particles have proven to be remarkably efficient scatterers; when evaluated in terms of the first hyperpolarizability $(\beta)$, HRS signals from the gold particles substantially surpass those observable from the best available molecular chromophores. Moreover, the present experiments indicate that $\beta$ is highly sensitive to colloid aggregation and imply that HRS is an effective tool for the characterization of symmetry-reducing perturbations of nanoscale interfaces.
\end{abstract}

The intentional miniaturization of metal/solution interfaces to the point where colloidal stabilization of suspensions of solvated metal particles occurs has been possible, at least at a rudimentary level, for more than a century. Arguably, however, only over the past decade has the concept generated widespread excitement among chemists and materials scientists. Fueling the contemporary interest are demonstrated and emerging applications involving medical screening, ${ }^{1}$ chemical sensing, ${ }^{2}$ singleelectron conduction (Coulomb blockade behavior), ${ }^{3}$ optical frequency tripling, ${ }^{4}$ and new materials-assembly schemes. ${ }^{5-7}$

Accompanying the new applications chemistry is a need for interface characterization. Among the more attractive methodologies for macroscopic metal/solution interface characterization is optical second harmonic generation $(\mathrm{SHG}){ }^{8}$ For otherwise centrosymmetric metal structures, interface formation creates an asymmetry providing for interface localized signal generation. SHG has been utilized to interrogate surface symmetry, ${ }^{9,10}$ surface charge, ${ }^{11}$ adsorbate coverage, ${ }^{12}$ and/or adsorbate orientation ${ }^{13}$ on gold surfaces as well as to interrogate gold particles at the liquid/air interface. ${ }^{14}$ However, the coherent nature of conventional SHG and the necessity of utilizing a finite wavelength of radiation place a practical lower limit on sample size. ${ }^{15}$ On the other hand, implementation of incoherent second harmonic generation or hyper-Rayleigh scattering (HRS) methods ${ }^{16}$ should circumvent the interface size limitation (albeit at the expense of much smaller signal intensity). ${ }^{17,18}$ On the basis of a successful application of HRS to nanoscale silica/water interfaces (surface acidity characterization) ${ }^{19}$ and on the basis of a brief report of HRS from silver colloid samples (molecular adsorption effects),${ }^{20}$ we reasoned that the methodology might be applicable to nanoscale gold/water interfaces, especially if plasmon enhancement of HRS were operative. We find not only that hyper-Rayleigh scattering is observable from aqueous metal (gold) nanoparticle suspensions but that the HRS signal intensities exceed by factors of up to $10^{5}$ those observed from suspensions of similarly sized wide band gap semiconductor or insulator particles. We additionally find that HRS is an exceedingly sensitive in situ reporter on nanoparticle aggregation and, more generally, on chemical or physical perturbations leading to particle and particle-assembly symmetry reduction.

For molecular systems (isotropic solutions), the HRS response, $I(2 \omega)$, is generally expressed in terms of an analyte hyperpolarizability, $\beta_{\mathrm{a}}$. Its value can be quantified by comparison to the HRS intensity of a solvent, or other internal reference, possessing a known first hyperpolarizability, $\beta_{\mathrm{s}} \cdot{ }^{21}$

$$
I(2 \omega)=G<N_{\mathrm{s}} \beta_{\mathrm{s}}^{2}+N_{\mathrm{a}} \beta_{\mathrm{a}}^{2}>I^{2}(\omega) \mathrm{e}^{\left(-N_{\mathrm{a}} \alpha_{\mathrm{a}} l\right)}
$$

In the equation, $G$ is a parameter reflecting collection efficiencies and local field corrections, $N$ is the number density $\left(\mathrm{cm}^{-3}\right)$ of the analyte or the solvent, $\alpha$ is the absorption cross section $\left(\mathrm{cm}^{-2}\right)$ at the scattered frequency $(2 \omega)$, and $l$ is the path length (cm) of the optical cell. The exponential term accounts for losses due to absorption of scattered light by the analyte solution, and the brackets signify that the measurement is an orientational average.

Figure 1 shows that output signal intensities at $410 \mathrm{~nm}$ from aqueous suspensions of nominally spherical, $13 \mathrm{~nm}$ diameter gold particles ${ }^{22}$ increase approximately as the square of the intensity of the incident light at $820 \mathrm{~nm}$, as expected if eq 1 were applicable. Also observed (Supporting Information and Figure 1) is a linear dependence of the $410 \mathrm{~nm}$ signal on gold particle concentration (number density) after correction for selfabsorption. A linear as opposed to quadratic $N_{\mathrm{a}}$ dependence implies that the doubled light is indeed due to HRS, rather than residual coherent SHG. ${ }^{15}$ A possible alternative assignment as two-photon fluorescence was eliminated by showing that the putative HRS signal is essentially monochromatic.

On the basis of $\beta_{\text {water }}=0.56 \times 10^{-30} \mathrm{esu},{ }^{23}$ eq 1 and Figure 1 yield a $\beta_{\text {gold }}$ value (on a "per atom" basis) of $(2800 \pm 500) \times$ $10^{-30}$ esu for incident light of $\lambda=820 \mathrm{~nm}$ and $(2000 \pm 300)$ $\times 10^{-30}$ esu for $\lambda=720 \mathrm{~nm}$. For comparison, $\beta$ values for the best molecular chromophores are on the order of $5000 \times 10^{-30}$ esu per molecule 24,25 or $(50-100) \times 10^{-30}$ esu per atom. As a further comparison, the frequency-doubling efficiency per atom of colloidal gold (proportional to $\beta^{2}$; see eq 1 ) is $10^{6}-10^{7}$ higher 


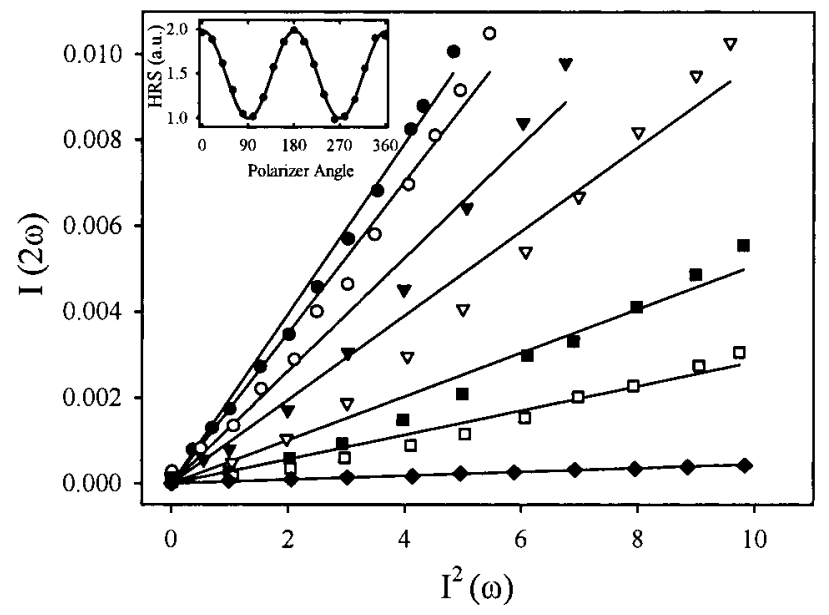

Figure 1. Power dependence of hyper-Rayleigh scattering, $\mathrm{I}(2 \omega)$, vs incident intensity squared, $P^{2}(\omega)$, for various concentrations of $\mathrm{Au}$ : $(\mathbf{0}$ $2.1 \times 10^{17} \mathrm{~cm}^{-3}$; (O) $8.4 \times 10^{16} \mathrm{~cm}^{-3} ;(\nabla) 4.2 \times 10^{16} \mathrm{~cm}^{-3} ;(\nabla) 2.1 \times$ $10^{16} \mathrm{~cm}^{-3} ;(\mathbf{\square}) 8.4 \times 10^{15} \mathrm{~cm}^{-3} ;(\square) 4.2 \times 10^{15} \mathrm{~cm}^{-3} ;(\diamond)$ water. Inset shows results of rotating a polarizer in front of the detector.

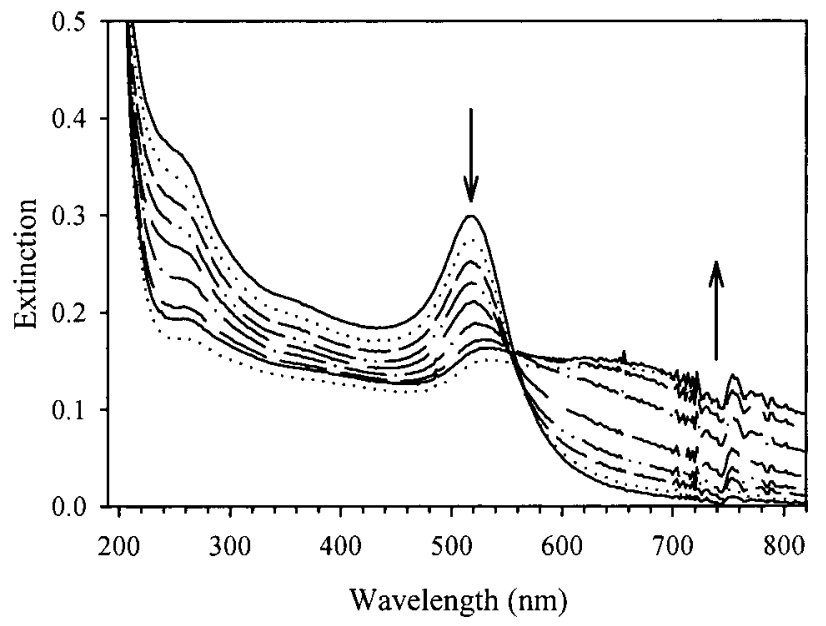

Figure 2. Extinction spectra recorded as $50 \mu \mathrm{L}$ aliquots of $6.0 \mathrm{M} \mathrm{NaCl}$ are added to $2 \mathrm{~mL}$ of the gold sol.

than reported for colloidal silver ${ }^{20}$ and $10^{3}-10^{5}$ higher than found for colloidal silica. ${ }^{19,26}$ We attribute the apparently unprecedented response to exceptionally effective resonance enhancement. ${ }^{27-29}$ In HRS, resonance enhancement is expected when an electronic transition is near either the incident $(\omega)$ or doubled $(2 \omega)$ frequency of light. ${ }^{30-32}$ Thus, the intense surface plasmon absorption $\left(\lambda_{\max }=520 \mathrm{~nm}\right.$; Figure 2$)$, known to enhance other spectroscopies, ${ }^{22,33}$ may lead to near resonance enhancement (both frequencies) in the HRS intensity. Alternatively, the enhancement may stem from two-photon resonance with the broad higher energy transition; planned experiments at longer wavelength should clarify the interpretation.

A further requirement for finite hyper-Rayleigh scattering is the absence of a symmetry inversion center-a requirement not fulfilled by an isolated metallic sphere. The observed scattering, therefore, is attributed to lower symmetry, nonspherical particles (isolated nanocrystals) or to lower symmetry aggregates of particles. In either case, only a small fraction of the available particles would likely be capable of HRS. To test the latter interpretation, irreversible particle aggregation was intentionally induced via addition of aqueous $\mathrm{NaCl}$. For substantial salt additions, the well-known red to blue colloid color change was directly observable. The change is due primarily to greatly increased Rayleigh scattering (one-photon scattering) in the red

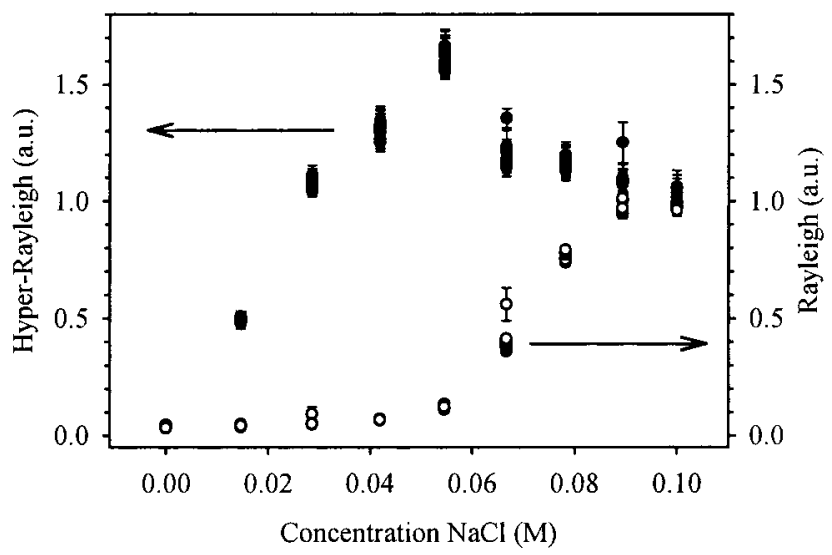

Figure 3. Effect of $\mathrm{NaCl}$ addition upon Rayleigh and hyper-Rayleigh scattering signal intensities. The scattering scales are arbitrary.

portion of the visible spectrum, due to large aggregate formation. ${ }^{34,35}$ As shown in Figure 3, the expected increase in Rayleigh scattering is almost unobservable when only small amounts of electrolyte $(\leq 30 \mathrm{mM})$ are added. In contrast, the intensity of hyper-Rayleigh scattering increases by at least an order of magnitude in response to the same solution compositional changes. ${ }^{36}$ We infer that HRS responds to much smaller aggregates than does Rayleigh scattering. Note that for a monodisperse collection of spheres, the smallest noncentrosymmetric assemblies would be nonlinear trimers (e.g., triangles) and compact tetramers (e.g., tetrahedra). We hope to evaluate hyper-Rayleigh scattering from intentionally prepared lowsymmetry trimers and tetramers and from related samples that lack centrosymmetry even at the single particle level.

Acknowledgment. We thank the Office of Naval Research, the Army Research Office (MURI program), and the Materials Research Center at Northwestern (NSF-DMR-9632472) for support of this work.

Supporting Information Available: Figure showing $N_{\mathrm{a}}$ dependence of $I(2 \omega)$ (1 page). Ordering information is given on any current masthead page.

\section{References and Notes}

(1) Storhoff, J. J.; Elghanian, R.; Mucic, R. C.; Mirkin, C. A.; Letsinger, R. L. J. Am. Chem. Soc. 1998, 120, 1959.

(2) Shaiu, W.-L.; Larson, D. D.; Vesenka, J.; Henderson, E. Nucl. Acids Res. 1993, 21, 99 .

(3) Andres, R. P.; Bein, T.; Dorogi, M.; Feng, S.; Henderson, J. I.; Kubiak, C. P.; Mahoney, W.; Osifchin, R. G.; Reifenberger, R. Science 1996, $272,1323$.

(4) See, for example the following. Hosoya, Y.; Suga, T.; Yanagawa, T.; Kurokawa, Y. J. Appl. Phys. 1997, 81, 1475.

(5) Schmid, G. Chem. Rev. 1992, 92, 1709 and references therein.

(6) Brust, M.; Bethell, D.; Schiffrin, D. J.; Kiely, C. J. Adv. Mater. 1995, 7, 795 .

(7) Feldheim, D. L.; Grabar, K. C.; Natan, M. J.; Mallouk, T. E. J. Am. Chem. Soc. 1996, 118, 7640.

(8) Corn, R. M.; Higgins, D. A. Chem. Rev. 1994, 94, 107 and references therein.

(9) Koos, D. A.; Richmond, G. L. J. Phys. Chem. 1992, 96, 3770.

(10) Yagi, I.; Nakabayashi, S.; Uosaki, K. J. Phys. Chem. B 1998, 102, 2677.

(11) Shi, Z.; Lipkowski, J.; Mirwald, S.; Pettinger, B. J. Electroanal. Chem. 1995, 396, 115.

(12) Jung, C.; Dannenberger, O.; Xu, Y.; Buck, M.; Grunze, M. Langmuir 1998, 14, 1103.

(13) Yang, D.; Bizzotto, D.; Lipkowski, J.; Pettinger, B.; Mirwald, S J. Phys. Chem. 1994, 98, 7083.

(14) Antoine, R.; Brevet, P. F.; Girault, H. H.; Bethell, D.; Schiffrin, D. J. Chem. Commun. 1997, 19, 1901. 
(15) It has been shown (Wang, H.; Yan, E. C. Y.; Borguet, E.; Eisenthal, K. B. Chem. Phys. Lett. 1996, 259, 15) that the limiting length scale can be at least an order of magnitude less than the wavelength of light.

(16) Clays, K.; Persoons, A. Rev. Sci. Instrum. 1994, 65, 2190.

(17) HRS arises from an instantaneous anisotropy that breaks the centrosymmetry present in an otherwise homogeneous solution.

(18) The orientation information present in SHG is also lost in an HRS experiment, where orientational averaging allows only for the reporting of the average symmetry of individual (noninteracting) chromophores.

(19) Vance, F. W.; Lemon, B. I.; Ekhoff, J. A.; Hupp, J. T. J. Phys. Chem. B 1998, 102, 1845.

(20) Clays, C.; Hendrickx, E.; Triest, M.; Persoons, A. J. Mol. Liq. 1995 $67,133$.

(21) Clays, K.; Persoons, A.; De Maeyer, L. Adv. Chem. Phys. 1994, 85 (III), 455.

(22) Grabar, K. C.; Freeman, R. G.; Hommer, M. B.; Natan, M. J. Anal. Chem. 1995, 67, 735 .

(23) Boutton, C.; Clays, K. Private communication.

(24) LeCours, S. M.; Guan, H. W.; DiMagno, S. G.; Wang, C. H.; Therien, M. J. J. Am. Chem. Soc. 1996, 118, 1497.

(25) Marder, S. R.; Cheng, L.-T.; Tiemann, B. G.; Friedli, A. C.; Blanchard-Desce, M.; Perry, J. W.; Skinhøj, J. Science 1994, 263, 511.

(26) Polarization experiments (Figure 1 inset) also reveal a dramatic difference between gold and silica colloids, where the ratio of vertically to horizontally scattered light for gold was found to be $2.2 \pm 0.2$ vs $22 \pm 3$ for silica (ref 19).

(27) The enhancement mechanism could be similar to that previously observed for hyper-Raman scattering experiments (refs 28 and 29), but differences in experimental conditions and parameters make a direct comparison difficult.

(28) Golab, J. T.; Sprague, J. R.; Carron, K. T.; Schatz, G. C.; Van Duyne, R. P. J. Chem. Phys. 1988, 88, 7942.

(29) Yang, W.; Hulteen, J.; Schatz, G. C.; Van Duyne, R. P. J. Chem. Phys. 1996, 104, 4313.

(30) A two-level model, derived for molecular chromophores, can be used to illustrate the energy dependence of $\beta$ on the electronic transition energy, $E_{\mathrm{op}}$, and the energy of incident radiation, $E_{\text {inc }}$ :

$$
\beta=\frac{3 \mu_{12}{ }^{2} \Delta \mu_{12} E_{\mathrm{op}}{ }^{2}}{2\left[E_{\mathrm{op}}{ }^{2}-E_{\mathrm{inc}}{ }^{2}\right]\left[E_{\mathrm{op}}{ }^{2}-\left(2 E_{\mathrm{inc}}\right)^{2}\right]}
$$

where $\mu_{12}$ is the transition dipole moment and $\Delta \mu_{12}$ is the change in dipole moment between ground and excited states.

(31) Oudar, J. L.; Chemla, D. S. J. Chem Phys. 1977, 66, 2664.

(32) Alternative two-level descriptions are also available. A full discourse on conventions for NLO can be found in the following. Willetts, A.; Rice, J. E.; Burland, D. M.; Shelton, D. P. J. Chem. Phys. 1992, 97, 7590.

(33) See, for example, the following. Frutos, A. G.; Corn, R. M. Anal. Chem. 1998, 70, 4494.

(34) Blatchford, C. G.; Campbell, J. R.; Creighton, J. A. Surf. Sci. 1982, $120,435$.

(35) Bohren, C. F.; Huffman, D. R. Absorption and Scattering of Light by Small Particles; Wiley: New York, 1983.

(36) $\beta$ (per atom) increases to ca. $8000 \times 10^{-30}$ esu. 